\title{
Hábitat y historia de vida de Pachychelles laevidactylus (Crustacea, Anomura, Porcellanidae) en el intermareal rocoso de Mar del Plata, Argentina
}

\author{
Martina Daniela Camiolo' \& Tomás Atilio Luppi²
}

\begin{abstract}
1. Instituto Nacional de Investigación y Desarrollo Pesquero (INIDEP), Consejo Nacional de Investigaciones Científicas y Técnicas (CONICET), Paseo Victoria Ocampo $n^{\circ}$ 1, CP 7600, Mar del Plata, Argentina.

2. Instituto de Investigaciones Marinas y Costeras (IIMyC), Consejo Nacional de Investigaciones Científicas y Técnicas (CONICET), Universidad Nacional de Mar del Plata, Funes 3350, CP 7600, Mar del Plata, Argentina.
\end{abstract}

Recibido 2 Julio 2015

Aceptado 25 Mayo 2016

DOI: $10.1590 / 1678-4766 e 2016015$

\begin{abstract}
Habitat and life's story of Pachychelles laevidactylus (Crustacea, Anomura, Porcellanidae) in the rocky shore of Mar del Plata, Argentina. Habitat structure, relative growth, the heterochely, population structure, morphometric maturity and fecundity of Pachycheles laevidactylus Ortmann, 1892 were analyzed during April, May and June 2005, in natural substrates of Mar del Plata, Argentina ( $38^{\circ} 02^{\prime}$ S, 57 $\left.7^{\circ} 31^{\prime} \mathrm{W}\right)$. Algal cover, wave exposure and immersion time varied significantly between strata and between months. Chelae morphometry showed no differences between sexes. Females showed the greatest abdomen. While there was the heteroquelia, laterality not detected. The size (carapace width) ranged from $0.67 \mathrm{~mm}$ to 9.3 $\mathrm{mm}$ for males and from $1.07 \mathrm{~mm}$ to $10.65 \mathrm{~mm}$, for females. The density of organisms varied among months and between strata. The population structure for the period of study explained depended on habitat structure. The morphometric maturity of females was recorded at the $4.38 \mathrm{~mm}$ of carapace width. The number of eggs was positively correlated with the size of the gravid females with a minimum of 2 eggs and a maximum of 160, but that relationship depended on progress in the development of embryos.
\end{abstract}

KEYWORDS. Life history traits, population structure, intertidal, morphometry.

RESUMEN. Se analizó la estructura del hábitat, el crecimiento relativo, la heteroquelia, la estructura poblacional, la madurez morfométrica y la fecundidad de Pachycheles laevidactylus Ortmann, 1892, durante Abril, Mayo y Junio del 2005, en sustratos naturales de Mar del Plata, Argentina (3802’S, $\left.57^{\circ} 31^{\prime} \mathrm{W}\right)$. La cobertura algal, la exposición al oleaje y el tiempo de inmersión varió de manera significativa entre estratos y entre meses. La morfometría de las quelas no manifestó diferencias entre sexos. Las hembras mostraron el abdomen más grande que los machos. Si bien existió la heteroquelia, no se detectó lateralidad. Las tallas (ancho de caparazón) estuvieron comprendidas entre $0.67 \mathrm{~mm}$ a $9.3 \mathrm{~mm}$ para los machos, y entre $1.07 \mathrm{~mm}$ a $10.65 \mathrm{~mm}$, para las hembras. La densidad de organismos varió entre meses y entre los estratos. La estructura poblacional, para el periodo de estudio, se explicó en función de la estructura del hábitat. La madurez morfométrica de las hembras fue registrada a los $4.38 \mathrm{~mm}$ de ancho de caparazón. El número de huevos se correlacionó positivamente con la talla de las hembras ovígeras con un mínimo de 2 huevos y un máximo de 160, pero esa relación dependió del avance en el desarrollo de los embriones.

PALABRAS-CLAVE. Rasgos de historia de vida, estructura poblacional, intermareal, morfometría.

Las especies que viven en el intermareal tienen adaptaciones específicas que les permiten contrarrestar el estrés asociado al ambiente (STILLMAN, 2002), el cual pueden ser de carácter regular, como las fluctuaciones de temperatura, salinidad y desecación, o estocásticos como la hidrodinámica turbulenta asociada a la rompiente y las tormentas marinas. Varios estudios han demostrado que cuanto mayores son los intervalos en que los sustratos están emergidos y sumergidos, más amplias son las variaciones térmicas; incluso, la tolerancia térmica puede ser un factor limitante más intenso que la desecación, especialmente cuando las temperaturas fluctúan en un periodo de tiempo muy corto (Jensen \& Armstrong, 1991; Stillman \& Somero, 2000). Además, la competencia, la predación y la preferencia por un tipo de sustrato también determinan el tipo de distribución (Stillman, 2002). En este sentido, muchos crustáceos viven asociados con especies sésiles (e.g. corales, moluscos, colonias de poliquetos y de urocordados y también con algas) y esta asociación puede brindar ciertas ventajas como obtención de refugio y alimento, o disminución de los efectos negativos de condiciones ambientales como la desecación, la temperatura o el efecto mecánico (JONES \& GREenWOOD, 1982; JeNSEN \& ARMSTRONG, 1991; SeIPLE \& Mueller, 1992; KNOTT et al., 2004; Yoder et al., 2005; EMPARANZA, 2007).

La historia de vida de un organismo se encuentra determinada por el conjunto de características que se relacionan de manera directa con la reproducción y la supervivencia, como así también con el tamaño y el patrón de crecimiento, la edad, la talla de maduración sexual, el 
número de descendientes y la longevidad (STEARNS, 1992). En los machos de cangrejos, la quela se considera la característica sexual secundaria más importante, con distintos niveles de alometría con respecto a las hembras y pueden aparecer fases de crecimiento a lo largo del desarrollo (HARTNOLL, 1974). La heteroquelia, la lateralidad y la diferencia de tamaños y crecimiento entre sexos se atribuyen a distintas características de la historia de vida de esos cangrejos como el tipo y tamaño de presa y, en el cortejo, la manipulación de hembras y en los encuentros agonísticos (ELNER, 1978; Abby-Kalio \& Warner, 1989; Lee, 1995). Por su parte, el pleon es la característica sexual secundaria más importante de las hembras y sobre la que clásicamente se realizan análisis morfométricos (HARTNOLL, 1974). La madurez sexual puede ser determinada de acuerdo con tres criterios: gonadal o fisiológica (madurez de esperma u oocitos), alométrica (medidas de los cambios en el crecimiento relativo de los caracteres sexuales secundarios), y funcional (capacidad de conseguir una pareja y transportar los huevos) (LóPEZ GReCo \& Rodríguez, 1998). En algunos casos la madurez gonadal ocurre antes que la madurez funcional. En otros es muy claro que la muda puberal puede ser asociada con los cambios en los caracteres sexuales secundarios en los juveniles (HARTNOLL, 1963, 1965).

Los porcelánidos son crustáceos anomuros de pequeño tamaño que viven en la franja costera, asociados a sustratos de naturaleza rocosa y a las diversas comunidades que allí se encuentran, siendo muchas veces los crustáceos más abundantes del intermareal (ANTEZANA et al., 1965; Ogawa \& Rocha, 1976; Scelzo, 1982; AlmaÇA, 1987; Pinheiro \& Fransozo, 1995; Stillman, 2002; EMParanza, 2007). Son animales de hábitos gregarios y no realizan desplazamientos extensos, excepto los que se producen durante la fase larval planctónica (AlmaÇA, 1987; PINHEIRO $\&$ Fransozo, 1995). Desempeñan un rol importante en la recuperación de la materia orgánica dado sus modos tróficos de filtradores y detritívoros (VIVIANI, 1969; SANHUEZA et al., 1975; GABALDON, 1979; Trager et al., 1992; ACHITUV \& PedrotTi, 1999); y cumplen un papel relevante en las tramas tróficas de las comunidades marinas (BosCHI et al., 1967; Ogawa \& Rocha, 1976; Scelzo, 1976; Cerda \& WolfF, 1993; Berríos \& VARGAS, 2004). El porcelánido Pachycheles laevidactylus Ortmann, 1892 se distribuye desde Pernambuco $\left(7^{\circ}-10^{\circ} \mathrm{S}\right.$, Brasil) hasta Mar del Plata y Monte Hermoso $\left(38^{\circ}-39^{\circ} \mathrm{S}\right.$, Argentina), incluyendo aguas tropicales, subtropicales y templadas (BosCHI, 1963, 1966; Scelzo, 2004). Es de hábitos costeros, se encuentra en el medio e infra litoral, ya sea en sustratos artificiales (M. Charrier Melillan \& M. A. Scelzo, datos no publicados) como naturales (BosCHI, 1966; Scelzo et al., 1996; PINHEIRO \& Fransozo, 1995) y se halla asociado principalmente al mejillinar formado por el mitílido Brachidontes rodriguezii (HaIG, 1966; Boschi et al., 1967; Scelzo et al., 1996).

Los trabajos realizados hasta el presente sobre esta especie constan de descripciones morfológicas del adulto y de las larvas (HAIG, 1966; BoschI, 1963; Boschi et al., 1967; M. Charrier Melillan \& M. A. Scelzo, datos no publicados), siendo escasos los antecedentes publicados sobre su historia de vida. Por este motivo los objetivos de este trabajo fueron: 1) describir la estructura del hábitat a la cual se encuentra asociada la especie, 2) estimar el crecimiento relativo de los caracteres sexuales secundarios y si existe preferencia bilateral en la heteroquelia, 3) establecer la estructura poblacional y 4) estimar la talla de madurez morfométrica, la asignación energética a la reproducción y la fecundidad.

\section{MATERIALES Y MÉTODOS}

Área de estudio. La investigación fue llevada a cabo en el intermareal rocoso de la ciudad de Mar del Plata, Argentina $\left(38^{\circ} 02^{\prime} \mathrm{S}, 57^{\circ} 31^{\prime} \mathrm{W}\right)$. Allí se encuentran los afloramientos rocosos más importantes de la costa bonaerense. Sobre estos afloramientos se desarrolla una comunidad intermareal de hasta 70 diferentes organismos, estructurada por especies sésiles como Brachidontes rodriguezii, Balanus amphitrite y B. trigonus (Olivier et al., 1966; SPIVAK et al., 1975; Scelzo, 1976; Elías \& VAllarino, 1997). Los muestreos se desarrollaron durante los meses de Abril, Mayo y Junio del 2005 (otoño) sobre cinco estratos diferentes con respecto a la altura y exposición al oleaje del mejillinar constituido por el mitilido Brachidontes rodriguezii; estratos 1 y 2 , superficies verticales y horizontales en el horizonte medio superior, estrato 3 , superficie horizontal en el horizonte medio medio, y estratos 4 y 5 , superficies verticales expuestas y protegidas al oleaje en el horizonte medio inferior (Horizontes según Olivier et al., 1966). Mediante la utilización de un sacabocados, de $20 \mathrm{~cm}$ de diámetro, se colectaron 5 muestras al azar (réplicas) en cada uno de los estratos.

\section{Estructura del hábitat}

I. Mejillinar. Se determinó la talla y la cantidad de mejillines. Para ello se extrajeron al azar 10 mejillines de 3 muestras de cada estrato y se les determinó el ancho y el largo de la valva. A su vez, se contabilizó la cantidad total de mejillines en cada una de las muestras de los 5 estratos estudiados y se determinó la densidad de cada uno de los mismos (individuos $* \mathrm{~cm}^{-2}$ ). Para estimar el espacio libre entre la parte inferior de los mejillines se calculó el espacio total (en una superficie equivalente al sacabocado de muestreo) correspondiente a la altura hasta el punto de contacto entre los laterales de los mejillines y se le restó el volumen correspondiente a la parte inferior del cuerpo del mejillín asumiéndolo como un cuerpo cónico. Además, se midió el volumen de arena de cada muestra para restárselo también al volumen disponible. El volumen disponible efectivo se expreso como porcentaje del volumen disponible teórico. También se registró la presencia de cuevas y sedimento debajo del mejillinar.

II. Cobertura algal. Se determinó la flora acompañante de los mitílidos. Las algas encontradas fueron clasificadas con el menor grado taxonómico posible, utilizando la clasificación de BOLD \& WynNE (1978). En todas las muestras analizadas de cada uno de los estratos se determinó el porcentaje de cobertura de cada especie de alga en la superficie del 
sacabocado de muestreo. Para evaluar si existieron diferencias significativas entre las proporciones de cobertura de cada alga entre los estratos se utilizó un ANOVA simple, y $a$ posteriori un test de Tuckey para identificar entre que estratos se manifestaron diferencias, previa transformación de los datos con arco seno de la raíz cuadrada de la proporción (ZAR, 1996).

III. Tiempo de inmersión. Se estimó el tiempo de inmersión del sustrato mediante observaciones de campo. Estas fueron realizadas durante 3 días no consecutivos en septiembre de 2005 (primavera), durante $6 \mathrm{hs}$, comenzando con la pleamar, y haciendo anotaciones a intervalos de media hora. El tiempo en el que cada estrato estuvo sumergido durante los cambios de marea fue considerado sobre el promedio de los 3 días de observación y luego los datos se extrapolaron a un periodo de $24 \mathrm{hs}$. Para evaluar si existieron diferencias entre las proporciones de los tiempos de inmersión entre los estratos se utilizó un ANOVA simple, y a posteriori un test de Tuckey para identificar entre que estratos hubo diferencias, previa transformación de los datos con arco seno de la raíz cuadrada de la proporción (ZAR, 1996).

IV. Temperatura Superficial del Mar. Los datos de Temperatura Superficial del Mar (TSM), correspondientes a Abril, Mayo y Junio de 2005, fueron obtenidos utilizando imágenes satelitales diarias del satélite-sensor Aqua-MODIS. Las mismas fueron descargadas de la página oficial de la NASA (http://oceancolor.gsfc.nasa.gov/cms/) en formato L2, con una resolución espacial de $1.1 \mathrm{~km}$. Posteriormente fueron procesadas con rutinas informáticas especificas y se obtuvo el valor de TSM promedio para cada mes y correspondiente desvío estándar. Las imágenes satelitales MODIS de TSM fueron validadas para el sur de la provincia de Buenos Aires (Argentina) y han demostrado un buen desempeño (Delgado et al., 2014).

\section{Características biológicas de la especie}

I. Morfometría. A cada uno de los individuos colectados se les determinó, el ancho del caparazón (AC), el largo de la quela, como el largo del propodus del primer pereiopodo, (LQ) tanto izquierda como derecha y el ancho del segmento mayor del pleon ( $\mathrm{AS}>$ ). El crecimiento relativo se evaluó por medio de rectas de regresión ajustadas a un modelo potencial. Como medida de referencia general se utilizó el AC. Para estimar si hubo diferencias significativas entre las pendientes de las regresiones se utilizó un ANCOVA, previa transformación de los datos con logaritmo en su base 10 (ZAR, 1996). El crecimiento alométrico se consideró isométrico cuando la pendiente tomó valores entre $0.9 \mathrm{y}$ 1.1 , positivo cuando la pendiente fue mayor a $1.1 \mathrm{y}$ negativo cuando fue menor a 0.9 (Clayton, 1990). Para estimar la talla de madurez morfométrica de las hembras se utilizó el método de Somerton (SomerTon, 1980).

II. Estructura poblacional. Los datos de AC se usaron para construir las distribuciones de frecuencias de tallas de cada período y estrato, con intervalos de clases de $0.25 ; 0.5$ y $1 \mathrm{~mm}$ a medida que las tallas aumentaron. Para descomponer en modas las distribuciones de frecuencias de tallas se utilizó el total de los datos de cada sexo. Las modas se estimaron usando el procedimiento MIX descripto por MACDonaLD \& PITCher (1979). La densidad de Pachycheles laevidactylus para cada estrato, mes y sexo se expresó como individuos cada $100 \mathrm{~cm}^{2}$ de mejillinar. La existencia de diferencias entre las densidades se analizó mediante un ANOVA de dos vías (ZAR, 1996), con las variables estratos y meses como factores. La proporción de sexos y de hembras ovígeras sobre el total de hembras, para cada mes y estrato se analizó mediante el análisis de Chi-cuadrado (ZAR, 1996).

III. Reproducción. Para determinar la fecundidad a cada hembra ovígera se contó el número de huevos y se la relacionó con el AC. Para determinar la asignación energética a la reproducción se utilizaron hembras ovígeras colectadas durante agosto y septiembre del 2005. A cada hembra se le contabilizó el número total de huevos, luego tanto la hembra como los huevos fueron secados en estufa siguiendo la metodología propuesta por LuPPI et al. (1997). El esfuerzo reproductivo fue estimado mediante el cociente del peso seco de la masa de huevos (solamente fueron considerados los huevos en estado I de desarrollo embrionario), sobre el peso seco de la hembra, y se lo relacionó con el AC de la hembra respectiva.

\section{RESULTADOS}

\section{Estructura del hábitat}

I. Mejillinar. Sobre los estratos 1 y 2 el largo medio de las valvas de los mejillines fue de $12 \pm 3.54 \mathrm{~mm}$ (Fig. 1). Sin espacio disponible bajo los mejillines debido a la presencia de gran cantidad de arena. En el estrato 3 el largo medio de las valvas de los mejillines fue de $15.4 \pm 3.84 \mathrm{~mm}$ (Fig. 1), y su densidad de 1.81 individuos $* \mathrm{~cm}^{-2}$. El espacio libre bajo el mejillinar fue del $27.1 \%$ del total teórico, reducido por la presencia de arena. Los mejillines estuvieron todos en contacto con el sustrato subyacente. En el estrato 4 el largo medio de las valvas de los mejillines registrada fue de $18.95 \pm 3.67 \mathrm{~mm}$ (Fig. 1), y la densidad de 1.74 individuos $* \mathrm{~cm}^{-2}$. El $100 \%$ del espacio entre los mejillines estuvo disponible, y no se registró arena bajo los mismos. Además, se evidenció la formación de cuevas y grandes espacios libres por debajo del mejillinar. Estas estructuras surgieron como consecuencia del crecimiento de mejillines sobre mejillines y porque partes del mejillinar no estuvo adherido al sustrato en su totalidad. Finalmente en el estrato 5 el largo medio de las valvas de los mejillines registrada fue de 22.1 $\pm 6.22 \mathrm{~mm}$ (Fig. 1) y la densidad de 1.74 individuo $* \mathrm{~cm}^{-2}$. La disponibilidad de espacios debajo de los mejillines fue del 100\%. La acumulación de arena entre los mejillines fue inexistente, y la formación de espacios entre y debajo de los mejillines aumentó con respecto al estrato 4.

II. Cobertura algal. En los estratos 1 y 2 no se detectaron algas. En el estrato 3 la flora acompañante estuvo compuesta principalmente por el alga verde Ulva rigida y por el alga roja Porphyra sp. Los porcentajes promedios de cobertura del sustrato fueron de $2.8 \pm 14.14 \%$ y de 16 $\pm 19.17 \%$ respectivamente. Los porcentajes de cobertura 


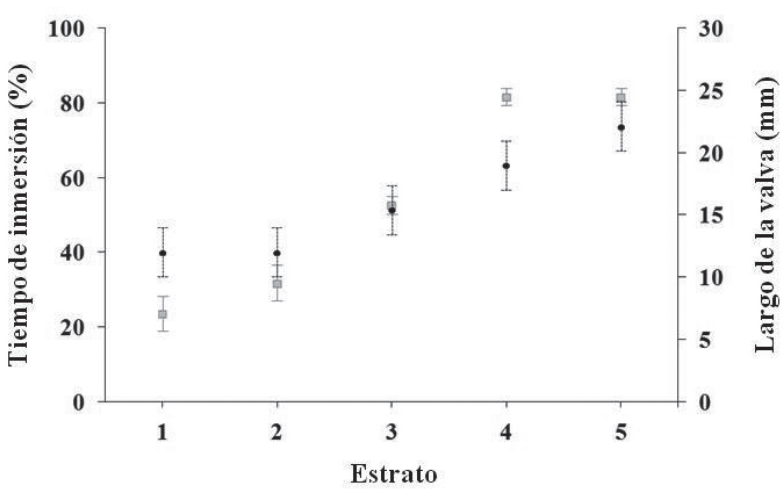

Fig 1. Tiempo de inmersión ( $\%$, cuadrados grises) para cada uno de los estratos y largo de la valva del mitílido Brachidontes rodriguezii (mm, círculos negros). Los cuadrados indican el promedio y las líneas verticales el desvío estándar.

tuvieron diferencias significativas entre los estratos para las dos especies $(\mathrm{F}=10.9, \mathrm{p}<0.002$ y $\mathrm{F}=9.2, \mathrm{p}<0.004$, respectivamente). En el estrato 4 la flora acompañante tuvo un porcentaje de cobertura de $29 \pm 19.49 \%$ para $U$. rigida (diferente al estrato $5, \mathrm{q}=5.6, \mathrm{p}<0.005$ ) y un $31 \pm 26.55$ $\%$ para Porphyra sp. (diferente al estrato $5, \mathrm{q}=4.08, \mathrm{p}<$ 0.034). Por su parte en el estrato 5 la flora acompañante estuvo dominada por Porphyra sp., con un porcentaje de cobertura del $68 \pm 8.36 \%$, en tanto que $U$. rigida cubrió un 3 $\pm 2.7 \%$ del sustrato. El porcentaje de cobertura de U. rigida fue diferente al del estrato $4(\mathrm{q}=5.8, \mathrm{p}<0.004)$, pero no al del 5 ( $q=0.19, p>0.99)$; en tanto que el de Porphyra sp. fue diferente al del estrato $5(\mathrm{q}=5.9, \mathrm{p}<0.003)$, pero no al del $4(\mathrm{q}=1.8, \mathrm{p}<0.41)$.

III. Tiempo de inmersión. En los estratos 1 y 2 el porcentaje de tiempo de inmersión del sustrato fue de 23.6 $\pm 4.6 \%$ y $31.8 \pm 4.8 \%$, respectivamente, siendo estos porcentajes significativamente diferentes a los de los otros sustratos $(\mathrm{F}=162, \mathrm{p}<0.001)$, pero iguales entre sí $(\mathrm{q}=4.01$, $\mathrm{p}>0.1$ ) (Fig. 1). En el estrato 3 el tiempo de inmersión fue del $52.6 \pm 2.3 \%$, y fue significativamente diferente a los estratos 1 y 2 , y 4 y $5(q=13.3, p<0.001$ y q $=9.3, p<0.001$, y q $=$ 13.8, $\mathrm{p}<0.001, \mathrm{q}=13.8, \mathrm{p}<0.001$ respectivamente) (Fig. 1). En el estrato 4 se encontró sumergido el $83 \%$ del total del tiempo, sin diferencias significativas con respecto al estrato 5 $(\mathrm{q}=0, \mathrm{p}=1)$ (Fig. 1); mientras que en el estrato 5 el tiempo de inmersión registrado fue igual que en el estrato 4 (Fig. 1).
IV. Temperatura Superficial del Mar. La temperatura media del agua en superficie obtenidas del satélite sensor Aqua-MODIS fue de $18.5 \pm 1.8{ }^{\circ} \mathrm{C}$ para el mes de Abril, $15.2 \pm 2{ }^{\circ} \mathrm{C}$ para el mes de Mayo y $13.5 \pm 1.6{ }^{\circ} \mathrm{C}$ para el mes de Junio.

\section{Características biológicas de la especie.}

I. Estructura poblacional. Fueron recolectados un total de 876 hembras y 677 machos de Pachycheles laevidactylus; hallados en los estratos 3,4 y 5 . No se registraron ejemplares en los estratos 1 y 2 durante el desarrollo de este trabajo. Para analizar la distribución de frecuencias de tallas mediante el programa Mix se utilizó el conjunto de individuos de las tres fechas de muestreo. Se identificaron 11 modas, tanto para machos como para hembras, con concordancias entre sexos en los valores estimados de los promedios de ancho de caparazón de cada moda (Fig. 2). La densidad de individuos estuvo comprendida entre los 2.5 individuos $* 100 \mathrm{~cm}^{-2}$ en el estrato 3 , y 14 individuos * $100 \mathrm{~cm}^{-2}$ en el estrato 5 . La cantidad de hembras y machos varió significativamente por mes y por estrato con interacción entre los factores para el caso de los machos (Tab. I). En las hembras, el estrato 5 fue el que mostró mayor presencia de todos los grupos de talla. Se observó la tendencia de una menor presencia de animales grandes en los estratos 3 y 4 . El estrato 3 tuvo muy pocos individuos. Este patrón de densidades decrecientes entre los estratos 5 a 3 se mantuvo en los tres meses muestreados también con un patrón decreciente entre abril y junio (Fig. 3). Los machos mostraron una tendencia similar, aunque más marcada. Fue más evidente la menor proporción de individuos grandes a medida que pasamos del estrato 5 al 3 , y en este último hubo muy pocos individuos. Sin embargo, las diferencias entre estratos fueron más importantes en mayo y junio (Fig. 3).

El patrón de proporción de sexos y de hembras ovígeras y no ovígeras varió de acuerdo al mes y estrato considerado. Durante el mes de Abril en los estratos 4 y 5 la proporción de hembras fue significativamente mayor $\left(\chi^{2}\right.$ $=4, \mathrm{p}<0.05$ y $\chi^{2}=11.47, \mathrm{p}<0.001$ respectivamente), que en el estrato 3. Por su parte, la proporción de hembras no ovígeras fue significativamente mayor en el estrato 3 y 5 $\left(\chi^{2}=6.12, \mathrm{p}<0.05\right.$ y $\chi^{2}=8.13, \mathrm{p}<0.01$ respectivamente $)$, pero no en el estrato 4. En el mes de Mayo la proporción de sexos no varió de la teórica 1:1 en los tres estratos, y la

Tab. I. ANOVA entre densidades de hembras y machos de Pachycheles laevidactylus Ortmann, 1892 con meses y estratos como factores y densidad de individuos como variable respuesta (DF, grados de libertad; SS, suma de cuadrados; MS, cuadrados medios).

\begin{tabular}{|c|c|c|c|c|c|c|}
\hline Sexo & Fuente de variación & DF & SS & MS & $\mathrm{F}$ & $\mathrm{P}$ \\
\hline \multirow[t]{5}{*}{ Hembras } & Mes & 2 & 1042.53 & 521.26 & 6.75 & 0.003 \\
\hline & Estrato & 2 & 8636.93 & 4318.46 & 5.96 & $<0.001$ \\
\hline & Mes $\mathrm{x}$ estrato & 4 & 463.73 & 115.93 & 1.5 & 0.222 \\
\hline & Residual & 36 & 2778 & 77.16 & & \\
\hline & Total & 44 & 12921.2 & 293.6 & & \\
\hline \multirow[t]{5}{*}{ Machos } & Mes & 2 & 762.31 & 381.15 & 9.65 & $<0.001$ \\
\hline & Estrato & 2 & 5173.51 & 2586.75 & 65.54 & $<0.001$ \\
\hline & Mes x estrato & 4 & 685.28 & 171.32 & 4.34 & 0.006 \\
\hline & Residual & 36 & 1420.8 & 39.46 & & \\
\hline & Total & 44 & 8041.91 & 182.77 & & \\
\hline
\end{tabular}




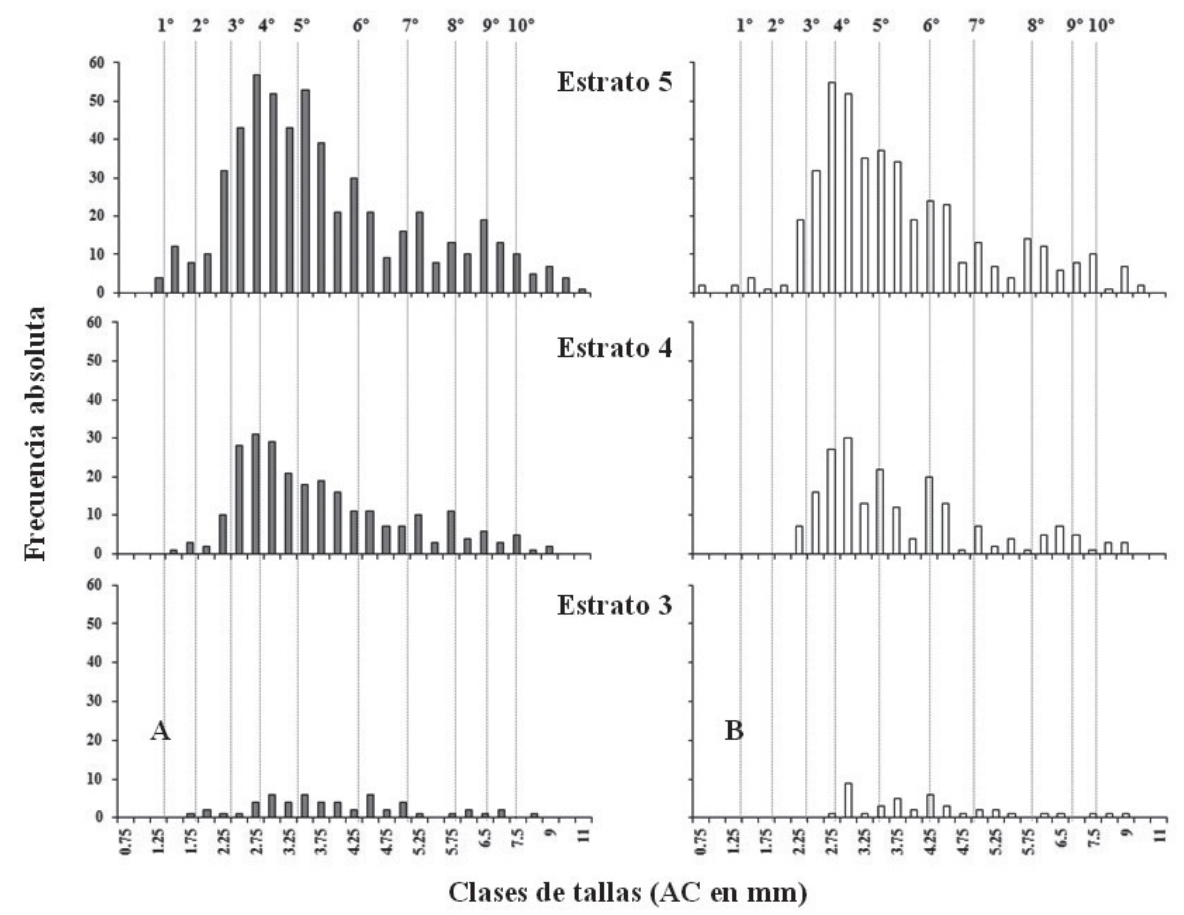

Fig 2. Pachycheles laevidactylus Ortmann, 1892: distribución de frecuencia de tallas de hembras (A) y machos (B) en los estratos 3 a 5. Las líneas verticales representan las medias de las modas calculadas.

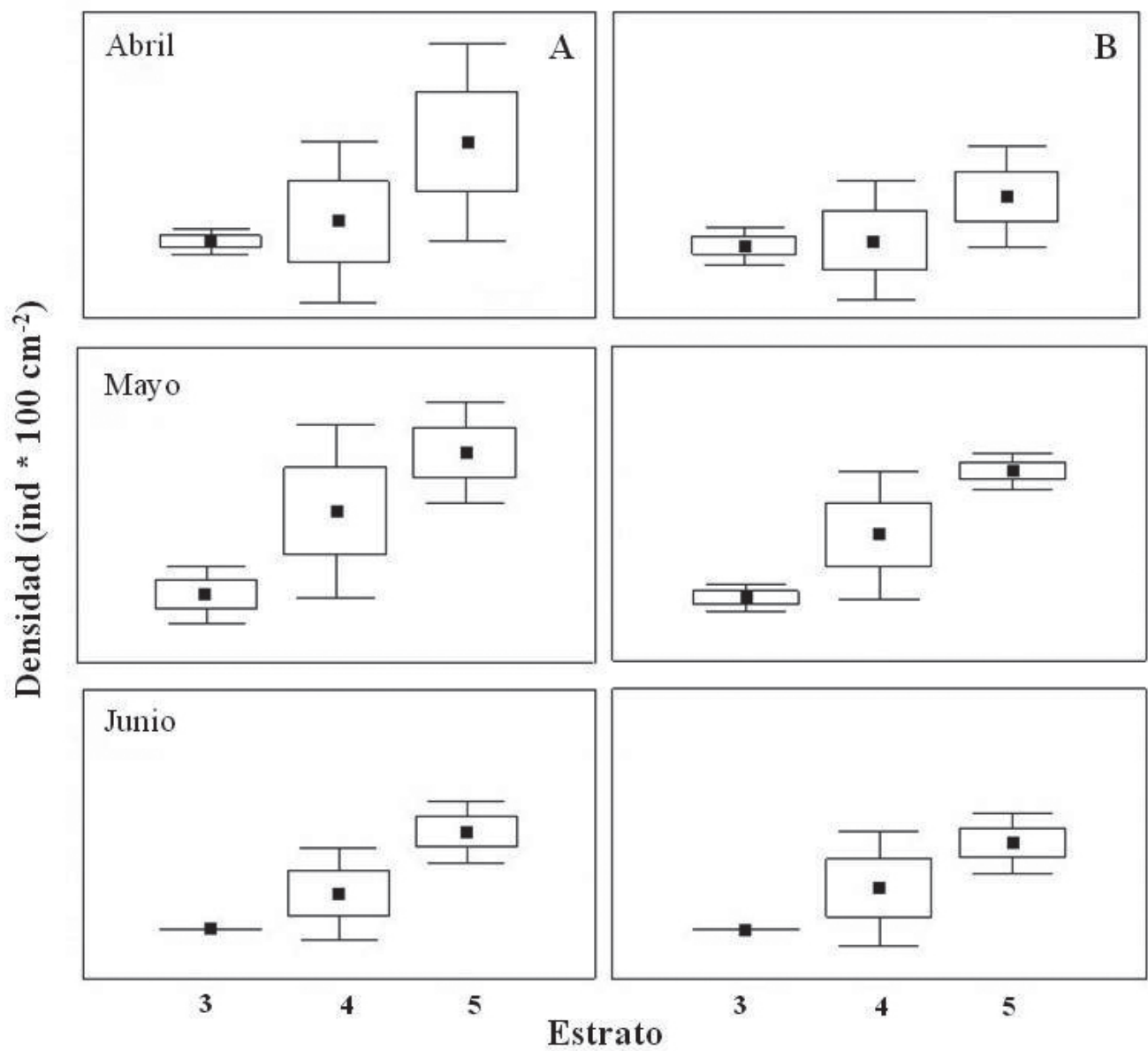

Fig 3. Densidad (individuos * $100 \mathrm{~cm}^{-2}$ ) de hembras (A) y machos (B) de Pachycheles laevidactylus Ortmann, 1892 en los estratos 3, 4 y 5, correspondiente al periodo de estudio. Los cuadrados indican el promedio y las líneas verticales el desvío estándar. 

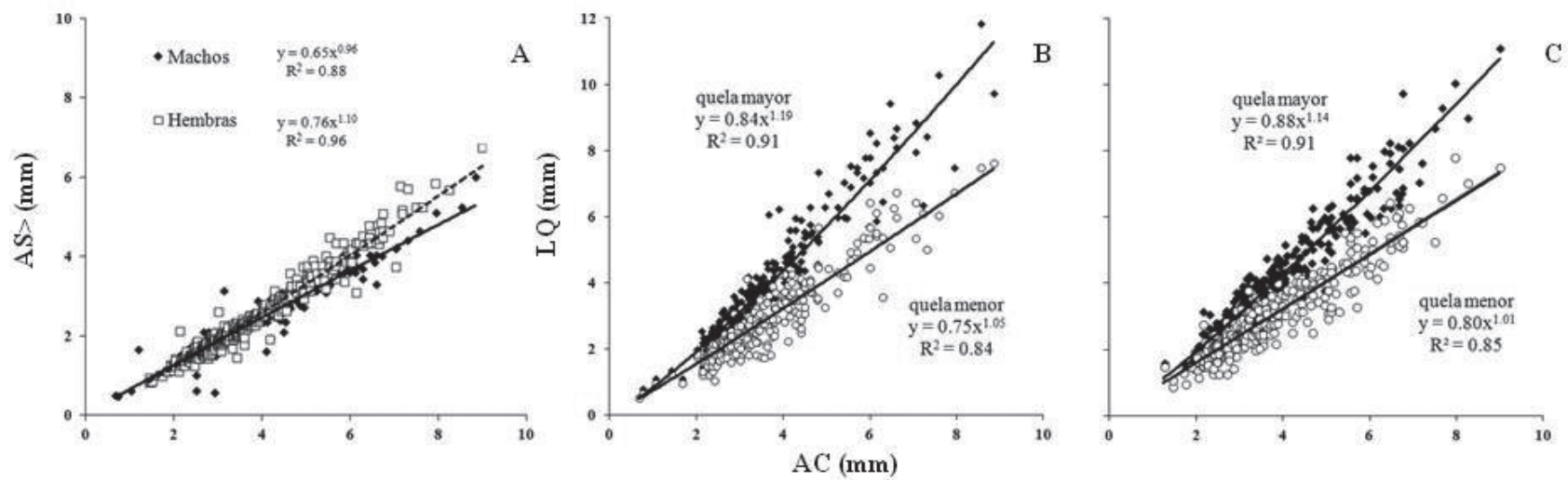

Fig 4. Relación del ancho de caparazón (AC) con respecto al segmento mayor del pleon (AS>) para ambos sexos (A), y su relación con el largo de la quela (LQ) mayor y menor para machos (B) y para hembras (C) de Pachycheles laevidactylus Ortmann, 1892.

proporción de hembras no ovígeras fue significativamente mayor en el estrato $5\left(\chi^{2}=3.95, \mathrm{p}<0.001\right)$. Durante el mes de Junio la proporción de sexos no varió de la teórica 1:1 en los estratos 4 y 5 . La proporción de hembras ovígeras fue significativamente mayor en el estrato $4\left(\chi^{2}=9.6, p<\right.$ 0.01 ), en tanto que en el estrato 5 lo fueron las hembras no ovígeras $\left(\chi^{2}=15.43, \mathrm{p}<0.001\right)$.

II. Morfometría. El AS $>$ de las hembras creció a una velocidad significativamente mayor en comparación al de los machos (Tab. II). El crecimiento en hembras fue alométricamente positivo, en tanto que en los machos fue isométrico (Fig. 4).

El LQ mostró diferencias significativas en su crecimiento relativo con respecto al AC cuando se compararon la quela mayor con la menor, tanto en machos como en hembras (Fig. 4). La quela mayor mostró un crecimiento alométrico positivo, mientras que en la quela menor el crecimiento fue isométrico en ambos sexos. No se detectaron diferencias significativas cuando se compararon las pendientes de las relaciones LQ con AC de quelas mayores y menores entre

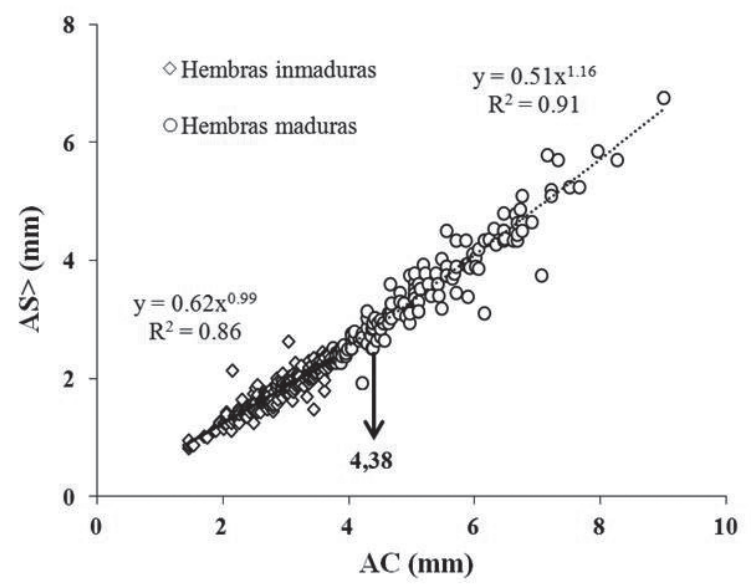

Fig 5. Crecimiento relativo del ancho del segmento mayor del pleon (AS $>$ ) con el ancho de caparazón (AC) en la fase inmadura y madura de Pachycheles laevidactylus Ortmann, 1892. La flecha indica la estimación de la talla de madurez morfométrica mediante el método de Somerton. sexos (Tab. II). La talla de madurez morfométrica se estimó en $4.38 \mathrm{~mm}$ de AC a partir del método de Somerton. Por debajo de este punto las hembras fueron morfométricamente inmaduras, y por encima morfométricamente maduras. Las relaciones entre $\mathrm{AS}>\mathrm{y} \mathrm{AC}$ de las hembras inmaduras y maduras tuvieron diferencias estadísticamente significativas en sus pendientes $(\mathrm{F}=17.5, \mathrm{p}<0.000)$. El crecimiento del pleon en las hembras inmaduras fue isométrico, en tanto que en las maduras fue alométricamente positivo (Fig. 5).

III. Reproducción. La fecundidad de las hembras de Pachycheles laevidactylus se incrementó conforme la talla aumentó y esta relación se vió representada de igual forma por un modelo exponencial o potencial. La fecundidad se acrecentó muy fuertemente en las tallas mayores y se mantuvo con valores muy bajos en las tallas menores. $\mathrm{Si}$ bien la talla de madurez morfométrica se estimó en 4.38 $\mathrm{mm}$ de $\mathrm{AC}$, hembras con tallas pequeñas cercanas a los 2 $\mathrm{mm}$ tuvieron huevos, aunque con valores que no superaron 2 huevos excepto un caso con 6 huevos (Fig. 6). La asignación energética a la reproducción se estimó en $5.03 \pm 1.94 \%$ de

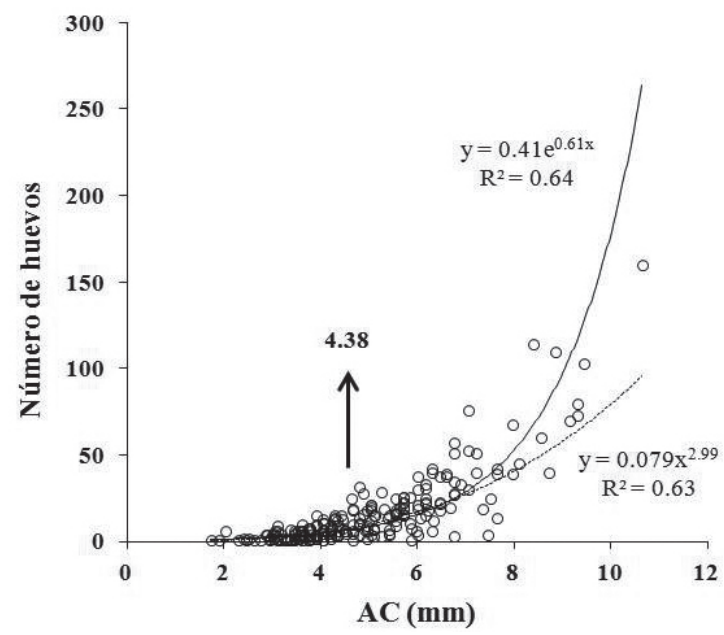

Fig 6. Relación de la fecundidad con el ancho de caparazón (AC) de Pachycheles laevidactylus Ortmann, 1892 explicado a través de un modelo exponencial y potencial. La flecha señala la talla de madurez morfométrica. 
Tab. II. Resultados de las comparaciones de pendientes realizadas con análisis de covarianza (ANCOVA) (AC, ancho de caparazón; F, estadístico del ANCOVA; $p$, probabilidad; *, indica cuando $\mathrm{p}<0,05)$.

\begin{tabular}{|c|c|c|c|}
\hline Variables & Comparación entre & $\mathrm{F}$ & $\mathrm{P}$ \\
\hline Ancho del segmento mayor del pleon & Sexos & 55.27 & $0.000^{*}$ \\
\hline Largo quela menor vs largo quela mayor & Sexos & 0.038 & 0.84 \\
\hline Alto quela mayor vs ancho caparazón & Sexos & 0.34 & 0.55 \\
\hline Alto quela menor vs ancho caparazón & Sexos & 1.37 & 0.24 \\
\hline
\end{tabular}

peso seco y no estuvo significativamente relacionada con el AC de las hembras.

\section{DISCUSIÓN}

Estructura del hábitat, distribución y estructura de talla. En la región costera de Mar del Plata, Pachycheles laevidactylus habita el mejillinar (Brachidontes rodriguezii) y ocupa de forma diferencial los distintos tipos de sustratos de acuerdo a su altura con respecto al agua, su orientación y su exposición al oleaje. Para el periodo de estudio, son varios los factores que pudieron intervenir en la determinación de este uso de hábitat diferencial. La desecación, como resultado directo del tiempo que permanecieron emergidos los sustratos más altos (estratos 1 y 2), surgió como el factor más importante para explicar la ausencia de la especie en esos estratos. El mismo argumento fue utilizado para explicar la ausencia de otros porcelánidos del genero Petrolisthes en sustratos que permanecieron más de 8 hs diarias al descubierto (Jones \& GREenwoOd, 1982; STILlMAN \& SOMERo, 1996). El espacio debajo de los mejjillines estuvo reducido por la presencia de arena. En el estrato 3 se encontraron individuos de Pachycheles laevidactylus en muy baja densidad. Los espacios bajo los mejillines fueron mayores, aunque la presencia de arena los redujo considerablemente, lo que limitó la talla y número de cangrejos presentes, en donde casi no estuvieron representadas las tallas mayores. PENCHASZADEH (1973) estimó que en los sustratos horizontales ocupados por mejillines podía acumularse hasta $19 \mathrm{~kg} \mathrm{~m}^{-2}$ de sedimento. El espacio libre entre los mejillines estuvo íntimamente relacionado con la abundancia de cangrejos del género Acanthocyclus (Brachyura) hallados en la costa rocosa de Chile (CAstilla et al., 1989), sin embargo, en Petrolisthes sp. se demostró que los sustratos con arena son evitados por el cangrejo, debido a su interferencia negativa en la actividad de alimentación de animales que son filtradores (JENSEN \& ARMSTRONG, 1991). La presencia de una importante cobertura de algas en este estrato pudo amortiguar los efectos negativos de la desecación y del estrés térmico. De hecho, frecuentemente fueron hallados individuos de P. laevidactylus agrupados debajo de acumulaciones de talos de Ulva rigida y Porphyra sp., y también dentro de conchas vacías del mejillín. En los estratos 4 y 5 se concentró la mayoría de los individuos colectados, ya que, el tiempo en que los sustratos estuvieron emergidos fue corto y prácticamente hizo desaparecer al shock térmico y a la desecación como limitantes en la distribución del cangrejo. Además, estos estratos, estuvieron rociados permanentemente por el oleaje. Los espacios debajo del mejillinar se incrementaron debido al aumento del tamaño de los mejillones y la ausencia de sedimento acumulado, con la consecuente expansión de la tridimensionalidad del sustrato. Este último factor tuvo gran importancia ya que permitió la presencia de individuos de tallas mayores a las de los espacios entre mejillinares debido al sobrecrecimiento de mejillines sobre otros y la aparición de capas de mejillines que no estuvieron completamente en contacto con el sustrato. Además, aumentó la cobertura de algas lo que mejoró aún más las condiciones de supervivencia del cangrejo. Sin embargo, hubo diferencias entre estos dos estratos. La densidad de individuos fue mayor en el estrato 5 que en el 4 y también fue mayor la presencia de hembras de tallas mayores. La exposición directa a la acción mecánica del oleaje que sufrió el estrato 4 pudo estar determinando estas diferencias, ya que ejerció una presión de disturbio sobre la comunidad, especialmente sobre la estabilidad de los refugios y también pudo hacerlo directamente sobre los cangrejos, o al menos parte de la población. En el estrato 5 se concentraron los individuos más pequeños de la población, lo que podría indicar una menor resistencia a la acción mecánica del oleaje. ELías et al. (1997) mostraron una correlación positiva entre la abundancia de P. laevidactylus y la talla de los mejillines $\mathrm{y}$, además, mostraron experimentalmente la preferencia por parte de los cangrejos de estratos cubiertos de refugios (valvas de mejillón o piedras) por sobre los descubiertos.

\section{Morfometría y reproducción.}

Pachycheles laevidactylus presentó heteroquelia para ambos sexos, pero no manifestó lateralidad. Prácticamente no se encontraron diferencias alométricas ni en la quela mayor ni en la menor entre sexos. Las quelas mayores crecieron a una velocidad mayor que las quelas menores $\mathrm{y}$, por lo tanto, las diferencias entre tamaños de las quelas aumenta en la medida que los cangrejos aumentan de talla. La alimentación de P. laevidactylus es por filtración, es decir no puede explicarse la heteroquelia y la lateralidad por los hábitos alimentarios, aunque esto no implicó que las quelas no pudieran cumplir un rol importante en la alimentación, tal como se ha descripto para otras especies de cangrejos porcelánidos (JENSEN \& ARMSTRONG, 1991). La no existencia de diferencias entre sexos también imposibilitó proponer explicaciones con base en sistemas de apareamiento, tales como que los machos poseen quelas más grandes para atraer las hembras o para contender con otros machos por la posesión de ellas. Sin embargo, es posible que la heteroquelia juegue un papel importante en la defensa de un recurso o en la competencia intra-específia, como ha sido observado en el cangrejo porcelánido Petrolisthes spinifrons (Baeza \& Asorey, 2012). BosCHI (1966) encontró lateralidad izquierda en P. laevidactylus, aunque incluía en su estudio individuos de 
mayor talla, lo que pudo ejercer influencia en los resultados. El pleon de las hembras de Pachycheles laevidactylus creció más rápidamente que el de los machos, especialmente en tallas superiores al punto de inflexión que indicó la madurez morfométrica de las hembras. La madurez morfométrica registrada para $P$. laevidactylus en este trabajo fue de 4.38 $\mathrm{mm}$ de $\mathrm{AC}$, la cual no coincidió con la madurez fisiológica encontrada, ya que se hallaron hembras ovígeras con un AC de $2.6 \mathrm{~mm}$. El mismo patrón se observó en el cangrejo braquiuro Neohelice granulata, la madurez gonadal ocurrió antes que la madurez alométrica (LóPEz GRECo \& RODRÍGUEZ, 1998). M. Charrier Melillan \& M. A. Scelzo (datos no publicados) registraron tallas muy similares para las hembras ovígeras, siendo estas de $2.5 \mathrm{~mm}$ de AC. Sin embargo, la talla mínima registrada en este trabajo fue menor que las encontradas por PinHeIRO \& Fransozo (1995) y por OgaWa \& Rocha (1976) en Brasil, siendo estas de $3.3 \mathrm{~mm}$ y $3 \mathrm{~mm}$ respectivamente.

El patrón de fecundidad de Pachycheles laevidactylus encontrado en sustratos naturales de Mar del Plata fue similar al de la misma especie encontrado en Ubatuba, Brasil (Pinheiro \& Fransozo, 1995), pero la fecundidad máxima fue sensiblemente menor. A las tallas de hembras máximas reportadas, la fecundidad máxima en Mar del Plata fue de 160 huevos en tanto que en Brasil fue de 775. Sin embargo, en otra población de Brasil, Fortaleza (Ogawa \& Rocha, 1976), la fecundidad máxima fue de 157 huevos. Muchos estudios en crustáceos sugieren un decrecimiento intraespecífico en la producción de huevos y en el esfuerzo reproductivo con el incremento de la latitud conjuntamente con un aumento en el tamaño de los huevos (Clarke \& Gore, 1992; Clarke, 1979; GoRNy et al., 1992). Esta hipótesis podría aplicarse para explicar parte de los resultados y comparaciones previas pero no la baja fecundidad reportada para Fortaleza, Brasil. Tampoco debe descartarse para el análisis las diferencias de sustrato en el que se desarrollan estas poblaciones. En Ubatuba, Brasil, los animales fueron obtenidos del interior de arrecifes de colonias del poliqueto Phragmatopoma lapidosa. Este ambiente brinda una protección muy grande en comparación con las superficies rocosas de Mar del Plata, tanto como de la acción del oleaje, como de la desecación y de los cambios de temperatura. La relación entre la fecundidad y la talla fue positiva en Pachycheles laevidactylus, al igual que en gran parte de los crustáceos (SASTRY, 1983). El modelo potencial al que se ajustaron los datos es uno de los modelos que más se utilizan en describir la relación entre número de huevos y talla en crustáceos. Para $P$. laevidactylus, se han encontrado poblaciones que responden mejor al modelo potencial (Ubatuba, Brasil, Pinheiro \& Fransozo, 1995) y otras al modelo lineal (Fortaleza, Brasil, Ogawa \& Rocha, 1976). Las hembras de P. laevidactylus tuvieron una asignación reproductiva de 5\% del peso seco. Este valor estuvo acorde a otros hallados para cangrejos porcelánidos como en los estudios de Lardies \& WeHrTManN (1996) y Hernáez \& Palma (2003) donde se registraron valores que oscilaron entre 3.7 y $7.4 \%$ en 6 especies de las costas chilenas, aunque también alcanzaron valores del $10 \%$ en Petrolisthes granulosus (LóPEZ et al., 1997). Los cangrejos porcelánidos tienen valores de asignación reproductiva por debajo del $10 \%$. La asignación reproductiva no tuvo una relación significativa con la talla de la hembra, similar a lo encontrado en otros cangrejos porcelánidos y braquiuros (LuPPI et al., 1997; HeRnáEZ \& PALMA, 2003).

\section{REFERENCIAS BIBLIOGRÁFICAS}

Abby-Kalio, N. J. \& Warner, G. F. 1989. Heterochely and handedness in the shore crab Carcinus maenas (L.) (Crustacea, Brachyura). Zoological Journal of the Linnean Society 96:19-26.

Achituv, Y. \& Pedrotti, M. L. 1999. Costs and gains of porcelain crab suspension feeding in different flow conditions. Marine Ecology Progress Series 184:161-169.

AlmaÇA, C. 1987. Crabs of the Sabellaria alveolata (Linnaeus, 1767) community. Egg number and population size in Pilumnus hirtellus (Linnaeus, 1761) and Porcelana platycheles (Pennant, 1777). Arquivos do Museu Bocage, Nova Série 1(3):19-32.

Antezana, T.; Faguetti, C. \& López, M. T. 1965. Observaciones bioecológicas en decápodos de Valparaíso. Revista de Biología Marina Valparaíso 12:1-60.

Berríos, V. \& VARGas, M. E. 2004. Estructura trófica de la asociación de peces intermareales de la costa rocosa del norte de Chile. Revista de Biología Tropical 52:201-212.

Bold, H. C. \& Wynne, M. J. 1978. Introduction to the algae. Englewood Cliffs, Prentice Hall. 706p.

Boschi, E. E. 1963. Sobre dos especies de Pachycheles de la Argentina (Crustacea, Anomura). Neotrópica 9(28):31-37.

Boschi, E. E. 1966. Preliminary note of the geographic distribution of the decapod crustaceans of the marine waters of Argentina (South-west Atlantic Ocean). Symposium Crustacea, India Part 1:449-456.

Boschi, E. E.; Scelzo, M. \& Goldstein, B. 1967. Desarrollo larval de dos especies de Crustáceos Decápodos en el laboratorio. Pachycheles haigae Rodrigues Da Costa (Porcellanidae) y Chasmagnathus granulata Dana (Grapsidae). Boletín del Instituto de Biología Marina de Mar del Plata 12:1-44.

Castilla, J. C.; Luxoro, C. \& Navarrette, S. A. 1989. Galleries of the crabs Acanthocyclus under intertidal mussel beds: their effects on the use of primary substratum. Revista Chilena de Historia Natural 62:199-204.

Cerda, G. \& Wolff, M. 1993. Feeding ecology of the crab Cancer polyodon in La Herradura Bay, northern Chile. II. Food spectrum and prey comsuption. Marine Ecology Progress Series 100:119-125.

Clarke, A. 1979. On living in cold water: K-strategies in Antartic benthos. Marine Biology 55:111-119.

Clarke, A. \& Gore, D. J. 1992. Egg size and composition in Ceratoserolis (Crustacea, Isopoda) from the Weddell Sea. Polar Biology 12:129-134.

Clayton, D. A. 1990. Crustacean allometric growth: a case for caution. Crustaceana 58:270-290.

Delgado, A; cédric, J.; Loisel, H.; Vantrepotte, V.; Perillo, G. \& Piccolo, M. C. 2014. Evaluation of the MODIS-Aqua Sea-Surface Temperature product in the inner and mid-shelves of southwest Buenos Aires Province, Argentina. International Journal of Remote Sensing 35(1):306-320

Elías, R. \& VAllarino, E. A. 1997. The dynamics o fan introduced Balanus glandula population in the South western Atlantic rocky shores. The consequences on the intertidalcommunity. PSZN. Marine Ecology 18(4):319-335.

Elías, R.; Vallarino, E. A.; Charrier, M. \& Gavio, A. 1997. Vertical distribution of the anomuran Pachycheles haigae and its relationship with the Brachydontes rodriguezi mussel beds in an intertidal rocky shore. VII Colacmar - Congresso Latino-Americano sobre Ciências do Mar 1:274-276.

ELner, R. 1978. The mechanics of predation by the shore crab, Carcinus maenas (L.) on the edible mussel, Mytilus edulis L. Oecologia 36:333344.

Emparanza, E. J. M. 2007. Patterns of distribution of dominant porcelain crabs (Decapoda, Porcellanidae). under boulders in the intertidal of northern Chile. Journal Marine Biology 87:523-531 
GABALDON, D. J. 1979. Observation on a possible alternate mode of feeding in a porcellanid crab (Petrolisthes cabrilloi Glassell, 1945) (Decapoda, Anomura). Crustaceana 36:110-112.

Gorny, M.; Arntz, W. E.; Clarke, A. \& Gore, D. J. 1992. Reproductive biology of caridean decapods from the Weddell Sea. Polar Biology 12:111-120.

HaIG, J. 1966. Résultats scientifiques des capagnes de la Calypso, fascicule 7. Campagne au large de côtes Atlantiques de l'Amérique du Sud (19611962). I. No. 2. Porcellanid crabs (Crustacea, Anomura). Annales de l'Institute Oceanographicque 44:351-358.

Hartnoll, R. G. 1963. The biology of Manx spider crabs. Proceedings of the Zoological Society of London 141:423-496.

Hartnoll, R. G. 1965. Notes on the marine grapsid crabs of Jamaica. Proceedings of the Linnean Society of London 176(2):113-147.

HARTNOLL, R. G. 1974. Variation in growth pattern between some secondary sexual caracters in crabs (Decapada, Brachyura). Crustaceana 27(2):131-136.

Hernáez, P. \& Palma, S. 2003. Fecundidad, volumen del huevo y rendimiento reproductivo de cinco especies de porcelanidos intermareales del norte de Chile (Decapoda, Porcellanidae). Investigaciones Marinas 31(2):35-46.

Jensen, G. C. \& Armstrong, D. A. 1991. Intertidal zonation among congeners: factors regulating distibution of porcelain crabs Petrolisthes spp. (Anomura, Porcellanidae). Marine Ecology Progress Series 73:47-60.

Jones, M. B. \& Greenwood, J. G. 1982. Water loss of a porcelain crab, Petrolisthes elongatus (Milne Edwards, 1837) (Decapoda, Anomura) during atmospheric exposure Comparative. Biochemistry Physiology 72a(4):631-636.

Knott, N. A.; Underwood, A. J.; Chapman, M. G. \& Glasby, T. M. 2004. Epibiota on vertical and horizontal surfaces on natural reefs and artificial structures. Journal Marine Biology 84:117-1130.

LARDiEs, M. \& WeHrTmanN, I. 1996. Aspects of the reproductive biology of Petrolisthes laevigatus (Guerin, 1835) (Decapoda, Anomura, Porcellanidae). Part I: Reproductive output and chemical composition of eggs during embryonic development. Archive of Fishery and Marine Research 43(2):121-135.

LEE, S. Y. 1995. Cheliped size and structure: the evolution of a multifunctiona decapod organ. Journal of Experimental Biology and Ecology 193:161-176.

LóPez, L.; Jeri, T.; GonzÁlez, C. \& RodríGuez, S. 1997. Fecundidad y esfuerzo reproductivo de Petrolisthes granulosus (Guérin, 1835) en Iquique, Chile (Decapoda, Anomura, Porcellanidae). Investigaciones Marinas 25:159-165.

López Greco, L. S. \& Rodríguez, M. 1998. Size at the onset of sexual maturity in Chasmagnathus granulata Dana, 1851 (Grapsidae, Sesarminae): a critical overall view about the usal criteria for its determination. In: Scharam, F. R. \& von VAupel Klein, J. eds. Crustacean and the Biodiversity crisis. Leiden, Brill, p. 675-689.

Luppi, T. A.; Bas, C. C.; SpIVACK, E. D. \& Anger, K. 1997. Fecundity of two grapsid species in the Laguna Mar Chiquita, Argentina. Archive of fishery and marine research 45(2):149-166.

MacDonald, P. D. \& Pitcher, T. J. 1979. Age-groups from size-frequency data: a versatile and efficient method of analysing distribution mixtures. Journal of Fisheries Research Board of Canada 36:987-1001.

Ogawa, E. F. \& Rocha, C. A. S. 1976. Sobre a fecundidade de crustáceos decápodos marinhos do estado do Ceará, Brasil. Arquivos de Ciências do Mar 16(2): 101-104.

Olivier, S. R.; Escofet, A.; Orensanz, J. M.; Pezzani, S. E.; Turro, A. M. \& Turro, M. E. 1966. Contribución al conocimiento de las comunidades bénticas de Mar del Plata. I: El litoral rocoso entre Playa
Grande y Playa Chica. Anuario Comisión Investigación Científica Provincia de Buenos Aires 7:185-206.

Penchaszadeh, P. E. 1973. Ecología de la comunidad del mejillín (Brachidontes rodriguezii, D’Orb.) en el mediolitoral rocoso de Mar del Plata (Argentina): el proceso de recolonización. Physis A 32(84):51-64. Pinheiro, M. A. \& Fransozo, A. 1995. Fecundidade de Pachycheles haigae Rodrigues da Costa, 1960 (Crustacea, Anomura, Porcellanidae) em Ubatuba (SP), Brasil. Revista Brasileira de Biologia 55(4):623-631.

Sanhueza, E. N.; Bahamonde, N. \& Lopez, M. T. 1975. Petrolisthes granulosus (Guerin) en biocenosis supramareales de El Tabo (Crustacea, Decapoda, Anomura). Boletin del Museo Nacional de Historia Natural 34:121-136.

SASTRY, A. N. 1983. Ecological aspects of reproduction. In: VERnBERG, F. J. \& Vernberg, W. B. eds. The Biology of Crustacea. Environmental adaptations. New York, Academic Press, p. 179-270.

Scelzo, M. A. 1976. Larvas de los Crustáceos Decápodos Anomuros identificadas en las aguas marinas argentinas. Physis A 35(90):37-45.

Scelzo, M. A. 1982. Observaciones sobre la presencia y distribución de crustáceos de la familia Porcellanidae (Decapada, Anomura) en aguas venezolanas y mar caribe. In: Zoología Neotropical. Actas del VIII Congreso Latinoamericano de Zoología. Tomo II, p. 1129-1146.

Scelzo, M. A. 2004. Cangrejos Anomura: Ermitaños y chinches de arena. In: Boschi, E. \& Cousseau, M. B. eds. La vida entre mareas: vegetales y animales de las costas de Mar del Plata, Argentina. Mar del Plata, Publicación especial INIDEP, p. 213-218

Scelzo, M. A.; Elías, R.; Vallarino, E.; Charrier, M.; Lucero, N. \& Alvarez, F. 1996. Variación estacional de la fauna acompañante del mejillín (Brachidontes rodriguezii) en Mar del Plata, Provincia de Buenos Aires, Argentina. Frente Marítimo 16:149-156.

Seiple, W. \& Mueller, B. 1992. Patterns of refuge use by Sesarma cinereum (Bosc). Bulletin of Marine Science 50(1):158-164.

Somerton, D. A. 1980. A computer technique for estimating the size of sexual maturity in crabs. Fisheries Aquatical Science 37:1488-1494.

SPIVAK, E.; BASTIDA, R.; L'Hoste, S. G. \& AdABBO, H. 1975. Los organismos incrustantes del puerto de Mar del Plata II. Biología y ecología de Balanus amphitrite y Balanus Trigonus (Crustacea, Cirripedia). LemitAnales 3:41-124.

Stearns, S. C. 1992. The evolution of life histories. Oxford, Oxford University Press. 264p.

Stillman, J. H. 2002. Causes and Consequences of Thermal Tolerance Limits in Rocky Intertidal Porcelain Crabs, Genus Petrolisthes. Integrative and Comparative Biology 42(4):790-796.

Stillman, J. H. 2000. A comparative analysis of upper thermal tolerance limits of eastern Pacific Porcelain crabs, genus Petrolisthes: Influences of latitude, vertical zonation, acclimation, and phylogeny. Physiological and Biochemical Zoology 73(2):200-208.

Stillman, J. H. \& Somero, G. N. 1996. Adaptation to temperature stress and aerial exposure in congeneric species of intertidal porcelain crabs (Genus Petrolisthes): correlation of physiology, biochemistry and morphology with vertical distribution. The Journal of Experimental Biology 199:1845-1855.

Trager, G. C.; Coughlin, D.; Genin, A.; Achituv, Y. \& Gangopadhyay, A. 1992. Foraging to the rhythm of ocean waves: porcelain crabs and barnacles synchronize feeding motions with flow oscillations. Journal of Experimental Marine Biology and Ecology 164:73-86.

Viviani, C. 1969. Los Porcellanidae (Crustacea, Anomura) chilenos. Studies on Neotropical Fauna and Environment 6:40-56.

Yoder, J. A.; Reinsel, K. A.; Welch, J. M.; Clifford, D. M. \& Rellinger, E. J. 2005. Herding limits water loss in the sand fiddler crab, Uca pugilator. Journal of Crustacean Biology 25(1):141-145.

ZAR, J. H. 1996. Bioestatistical analysis. 3ed. Upper Saddle River, New Jersey, Prentice Hall. 662p. 